\title{
A Novel Approach to Assist Faculties In Question Paper Generation
}

\author{
D. Radha \\ M.O.P Vaishnav College \\ For Women \\ Chennai, India
}

\author{
R. Jayaparvathy \\ S.S.N College of Engineering \\ Chennai,India
}

\author{
Bavadaarane M. \\ M.O.P Vaishnav College \\ For Women \\ Chennai, India
}

\author{
A. Jyothirmayi Bhargavi \\ M.O.P Vaishnav College \\ For Women \\ Chennai, India
}

\begin{abstract}
Professors' feel arduous to design questions for an examination. It is quite obvious that even experienced educators face difficulty to frame question papers within short span of time. This paper concentrates to device an Intelligent Mathematical model to reduce the time dedicated to generate question papers. 'Knowledge Discovery in Database', is the decisive role of Data Mining that adopts to transform raw data into constructive information from large data repositories, and so it is deployed to achieve the objectives.
\end{abstract}

\section{General Terms}

Mathematical model, Knowledge Discovery in Database, Data Mining, Repositories, Extraction

\section{Keywords}

Question paper generation, Mathematical model, Stemming, Filtering

\section{INTRODUCTION}

Universities proffer voluminous Undergraduate (UG) and Postgraduate (PG) courses. The most tedious chore is to frame question papers on a peculiar domain. Consequently professors' has to possess requisite knowledge, perhaps tutors need to scrutinize the whole book to generate question. Suitably, this proposed system recommends mentors to provoke interrogation. This progression facilitates to enhance the quality of a question paper. Based on logic, length, diagrammatic representation and importance, questions can be framed from easy to tough and vice versa. Furthermore these features lay as a milestone to access quick question papers to overcome time constraint. As an outcome this assists lecturers to develop questions from any complicated textbook using the proposed technique. Knowledge Discovery and Data Mining target on the technique of abstracting crucial data. Data mining is a manner to refer useful concept and study information from disparate angle and encapsulate it to advantageous information. This mechanism axiomatically excerpts knowledge. Predictive analysis is a technique that handles earlier data to construct an automatic predictive model for certain applications. A collective experience can be gained by weighing the extant attributes. Classification technique is used to analyze the data. It is a task where a model or classifier is built to predict class labels. It comprises of two-phases; learning and a classification. The learning phase focuses on developing a classification model. The second phase comprises of predicting class labels for the given data.

\section{LITERATURE REVIEW}

"An Overview of Automatic Question Generation Systems"- Deepshree S. Vibhandik, and Prof. Mrs. Rucha C. Samant ${ }^{[1]}$ proposed an Automatic Question Generation (AQG) to generate reasonable questions from an input, which can be structured (e.g. a database) or unstructured (e.g. a text). AQG can be a good tool to help and to improve the quality of the Question Answering (QA) systems. This framework helps in question generation and the agents will be allowed to perform various operations like document processing, information classification and question generation.

"Automatic Generation of Question Answer Pairs from Noisy Case Logs" - J.Ajmera, S,Joshi, A.Verma, A.Mittal ${ }^{[2]}$. Proposed a technique to exploit the case logs by concerns problems and map it to related article and these mapping results into generation of question-answer (QA) pairs. Finally 'Crisp problem - Statement' is deceived. This is achieved by a composite model consisting of two generative models, viz, hidden Markov model (HMM) and latent Dirichlet allocation (LDA) model.

"Automatic Question Generation using Software Agents for Technical Institutions" - Shivank Pandey and K.C. Rajeswari ${ }^{[3]}$. Focused on the text file from user's which contains text, upon which user desires to fetch questions. The output is produced in form of a text file containing questions based on Bloom's taxonomy. The entire process is carried out by software agents, which eliminates the major problems.

"Modeling an Agent for Paper Generation System using Utility Based Approach" - M. Junaid Arshad , Mamoona Naz , Yasir Saleem , Amjad Farooq , K.H.Asif. ${ }^{[4]}$ The proposed system automates test paper generation using multi-agent approach takes into account the difficulty level of questions and the type. This technique is based on approximation of fitness value and probabilities of survival of solution i.e. test paper. The multi-agent paradigm abates issues in conventional client server approach.

"Utility based Agent for question paper generation" Memoona Naz, M. Aslam, Ehtesham-ul-haq Dar in $2010^{[5]}$, proposed the difficulty level of questions while generating question paper, its prerequisite questions in the 
database, from which the agent selects questions according to the difficulty level determined by the examiner.

\section{SYSTEM MODEL}

\subsection{Description for Each Value of an Attribute}

The classification technique is used to predict the difficulty rate of a particular question. In this work, the data set is established in the form a table consisting of rows and columns where rows correspond to the values for each attribute of an object and columns represents the attributes. The attributes are question, logic, length, diagrammatic representation and importance. The target variable is the Difficulty Rate $(D R)$ attribute which determines difficulty of the question.

The value of logic attribute can range from $1-3$. The length attribute's value can range from 1-3. The value of diagrammatic representation, importance can range from 0-1, 1-3 respectively (Refer Table: 1, 2, 3, 4). The value of each attribute of an object is summed up and is assigned to the total attribute.

Meaning for each value of an attribute is given below.

Table 1. Logic: Represents the difficulty rate of the question

\begin{tabular}{|c|c|}
\hline 1 & Easy \\
\hline 2 & Medium \\
\hline 3 & Difficult \\
\hline
\end{tabular}

Table 2. Length: Represents the length of the answer

\begin{tabular}{|c|c|}
\hline 1 & Short \\
\hline 2 & Lengthy \\
\hline 3 & Very lengthy \\
\hline
\end{tabular}

Table 3. Diagrammatic Representation: Represents diagrammatic supports to answer

\begin{tabular}{|c|c|}
\hline 0 & Diagram is not needed \\
\hline 1 & Diagram is a must \\
\hline
\end{tabular}

Table 4. Importance: how important the question is from the exam point of view

\begin{tabular}{|c|c|}
\hline 1 & Less important \\
\hline 2 & Medium \\
\hline 3 & Very important \\
\hline
\end{tabular}

\subsection{Formula Description}

Total $=\sum^{n} V_{i}$ $i=1$

' $n$ ' refers to the number of attributes, ' $\mathrm{Vi}$ ' refers to the value of the attribute

\section{PERFORMANCE AND ANALYSIS}

The attributes question, logic, length, diagrammatic representation and importance are considered to find the difficulty rate of the question. The abbreviations are used in this technique for the attributes.

Table 5. Acronym

\begin{tabular}{|c|c|}
\hline QUE & Question \\
\hline LOG & Logic \\
\hline LEN & Length \\
\hline DR & Difficulty Rate \\
\hline IMP & Importance \\
\hline TOT & Total \\
\hline
\end{tabular}

Table 6. Subset of the Data Set

\begin{tabular}{|c|c|c|c|c|c|c|}
\hline QUE & LOG & LEN & DR & IMP & TOT & Mode \\
\hline A & 3 & 3 & 1 & 2 & 9 & Difficult \\
\hline B & 2 & 2 & 1 & 2 & 7 & Moderate \\
\hline C & 1 & 3 & 1 & 1 & 6 & Moderate \\
\hline D & 2 & 3 & 1 & 1 & 7 & Moderate \\
\hline E & 2 & 1 & 1 & 1 & 5 & Moderate \\
\hline F & 2 & 3 & 1 & 1 & 7 & Moderate \\
\hline G & 1 & 3 & 0 & 2 & 6 & Moderate \\
\hline H & 2 & 3 & 1 & 3 & 9 & Difficult \\
\hline I & 3 & 2 & 1 & 1 & 7 & Moderate \\
\hline J & 3 & 1 & 0 & 2 & 6 & Moderate \\
\hline
\end{tabular}

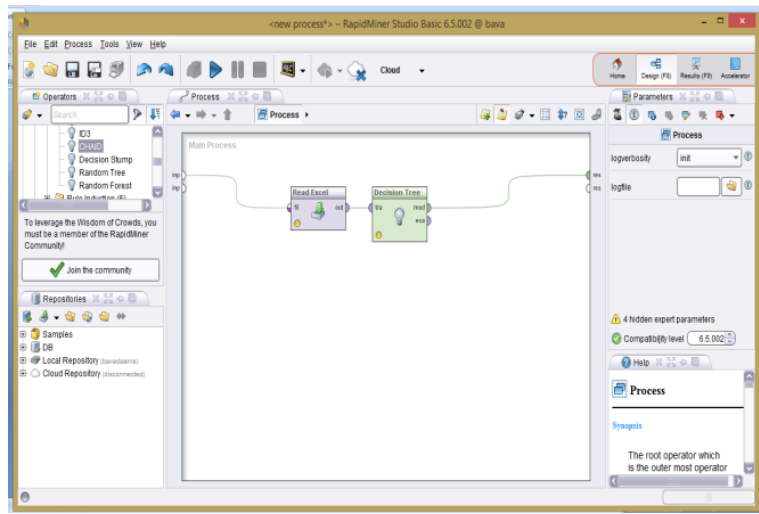

Figure (1) shows the results of deploying Rapid miner data mining tool to generate the decision tree 


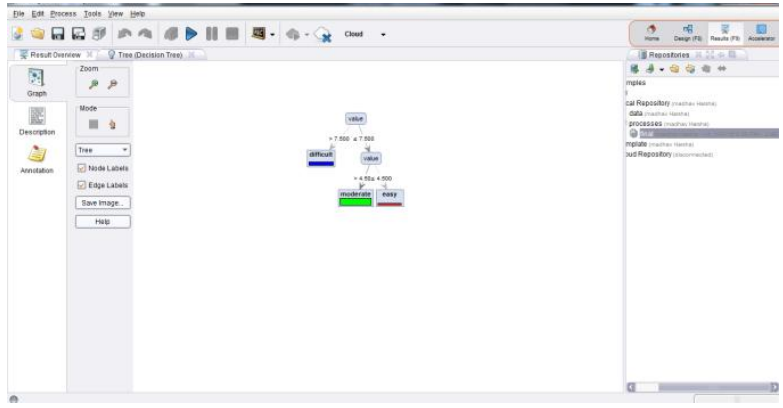

Figure (2) shows the results of deploying Rapid miner data mining tool to generate the decision tree.

If the value of total attribute is greater than 7.5, the question is difficult; if it is greater than 4.5 and less than 7.5 , the question has a moderate difficulty and if the value is less than or equal to 4.5 , the question is easy. The above model is evaluated for 1000 records. Accuracy of the above proposed model was found to be $95 \%$.

\section{CONCLUSION AND FUTURE ENHANCEMENTS}

This research work emphasis on making the task of generating question papers easier. The efficiency of the proposed model is observed to be $95 \%$. The research work aids in generating questions based on the difficulty rate Questions will be segmented not only based on their difficulty rate but also on grade. This development can make the process of generating question papers even easier.

\section{REFERENCES}

[1] "An Overview of Automatic Question Generation Systems" - Ms. Deepshree S. Vibhandik, Prof. Mrs. Rucha C. Samant. International Journal of Science, Engineering and Technology Research (IJSETR), Volume 3, Issue 10, October 2014

[2] "Automatic Generation of Question Answer Pairs from Noisy Case Logs" - J.Ajmera, S,Joshi, A.Verma, A.Mittal. Data Engineering (ICDE), 2014 IEEE 30th International Conference on March 31 2014-April 42014
[3] "Automatic Question Generation Using Software Agents for Technical Institutions" - Shivank Pandey, K.C. Rajeswari. International Journal of Advanced Computer Research [ISSN: 2249-7277] Volume-3 Number-4 Issue-13 December-2013

[4] "Modeling an agent for Paper Generation System using Utility Based Approach" - M. Junaid Arshad , Mamoona Naz, Yasir Saleem, Amjad Farooq, K. H. Asif . Journal of Faculty of Engineering \& Technology journal homepage JFET 19 (2012) 109124

[5] "Utility Based Agent for Test Paper Generation " Memoona Naz, M. Aslam, Ehtesham-ul-haq Dar. International Journal of Multidisciplinary Sciences and Engineering, Vol. 1, No. 1, September 2010 [ISSN: 2045-7057]

[6] "Framework for Automatic Examination Paper Generation System" - Ashok Immanuel, 2Tulasi.B Christ University, Bengaluru, Karnataka, India. IJCST Vol. 6, Issue 1, Jan - March 2015

[7] "Automatic Question Paper on generation System using Randomization algorithm" - Kapi; Naik, Shreyas Sule, Shruthi Jadhav, Surya Pandey International Journal of Engineering and Technical Research (IJETR)ISSN:2321-0869, Volume-2, Issue12, December 2014

[8] "Intelligent Question Paper Generator System" Hkda-Iqps - Standardization of Intelligent Tutoring System for Adaptive Information Retrieval and Knowledge Discovery by Monitoring Human Interaction

[9] "Detailed Project Report (DPR) On Automation of Question Paper Preparation" - Anil Kumar, VR Satyabrat Das - Indian Institute of Information Technology and Management, Kerala IIITM, Techno park, June 2013

[10] "Reflecting on Good Teaching" - Chatterjee.D, $\operatorname{IJES} 3(2)$, 